\title{
DATA ANALYTICS APPROACH FOR HEALTH MONITORING USING WEARABLE SENSOR
}

\author{
S.DILIP KUMAR ${ }^{1}$, P.MANJU $^{2}$, R. NAGALAKSHMI ${ }^{3}$, R.M. NANDHINI ${ }^{4}$, B. SANGAVI $^{5}, \mathrm{~S}$ AGASH $^{6}$ \\ ${ }^{1}$ Assistant Professor, ${ }^{2}$ Professor, ${ }^{3,4,5,6}$ Students \\ 1,2,3,4,5,6 Instrumentation and Control Engineering Sri Krishna College of Technology, Coimbatore
}

\begin{abstract}
In this age of technological advancement, individuals are becoming less involved incompletion their physical work. Tele medicine plays an important role in the new trend, promoting the transition of health care services from clinic-centered to patient-centered with the aid of omnipresent social connectivity. One of the applications facilitated by data analysis is the continuous health management frame work. Wearable sensor devices used in Data Analytics have continuously produced an enormous amount of data for the health monitoring system. The speed of data generation for data analytics equipment is very high, so the amount of data generated from it is also high. In order to track critical parameters such as blood pressure, body temperature, pulse rate, the sensor is integrated with humans. The approach of data analytics compares the health parameter of the person and, if necessary, makes a decision. This approach provides the healthcare provider with a quick but powerful and reliable communication tool to have the solution in hand before the situation gets worse. Proteus professional software has been used to execute the concept.
\end{abstract}

Keywords: Data analytics, Wearable sensor, Health monitoring, Proteus professional.

\section{Introduction}

Happiness is commensurate with the wellbeing of the person. Without well being, no happiness can be achieved. In order to live more comfortably, health is important. But health-related illnesses, on the other hand, the grow than advance mention science and technology is rising day by day [1]. Science and technology are in search of Health-relate disuses control. The increased use of health-related mobile

technology and smart devices have a huge impact on people. A health monitoring system using IOT is proposed where the authorized personal can access these data stored using any IOT platform and based on the values received, diseases are diagnosed by the doctors for much distance [2].

Through the design of Data analytics approach for health Monitoring system, we can monitor heartbeat rate, temperature and pressure of people with different age groups. This paper is designed to monitor the heart beat of a person by using heart beat sensor, monitor the temperature of the body by using temperature sensor LM35 and blood pressure using MPX4115. For people far away from cities and living in villages, the health monitoring system may prove more useful. Such individuals are unable to enter the hospital in good time to save their life. By planning such, together with E-mail warning and storing data in cloud which can easily save the patient's life by attaching the health monitoring system to the patient's body, and they can easily be diagnosed online through the internet. So, we introduced a method which continuously monitors the patient's condition and automatically transmit the data to server, so the doctor can access the data continuously and we can intimate caretaker when patient is in critical situation [3].

When a person's health problem has progressed to the point that his or her life is in jeopardy, they seek medical help, which may result in an unnecessary waste of money. By offering online medication according to the health condition, a doctor may diagnose the patient in order to hospitalize the patient on time if necessary, so that the risk of losing patient life can be reduced.

\section{PROPOSEDWORK:}

The main aim of our project is to make the frame work for health monitoring simple and precise. Health monitoring system include the collection and processing of data obtained from smart phones, smart watches, smart bracelets, as well as from various age groups of people with different sensors. Using a data analytics approach, a health monitoring device can measure the pulse rate, temperature, and blood pressure of patients of any age group, and these data can then be translated into a patient's qualified data collection. These data can also be sent to health care centers which will also have the knowledge about patient's health. By this when our health condition varies health care center will contact us to take preventive measures. All of these parameters can be transmitted to the Blynk Cloud wirelessly. Test values can be transmitted wirelessly to physicians in emergency situations and the patient will also be alert using IOT via E-mail and blynk app. In our design we have integrated all three parameters in a single system to make it cost-effective. All the data which 
have been stored in cloud can be accessed or extracted at any time to understand the health status of the patient.

\section{BLOCKDIAGRAM}

Fig.3.1shows the block diagram of health monitoring system and its working.

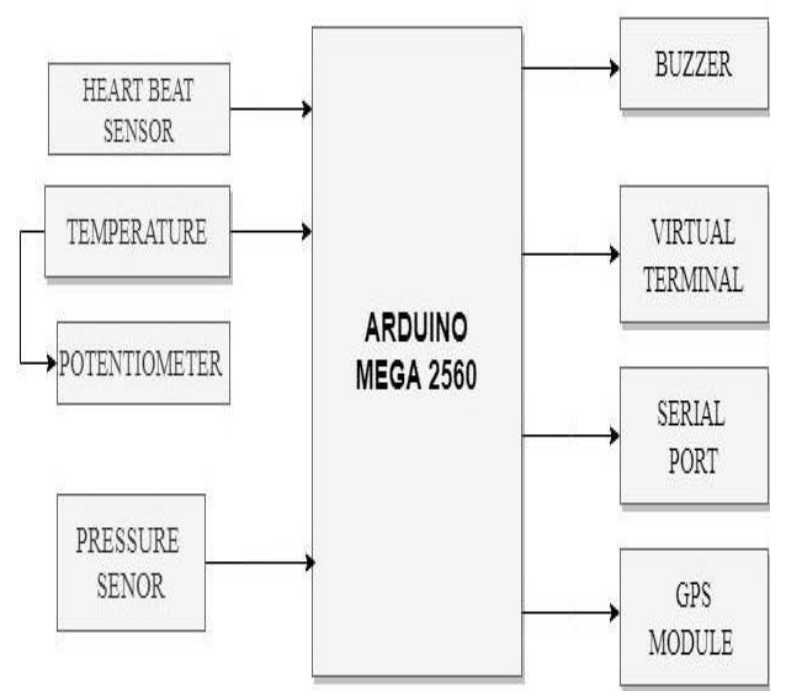

Fig3.1.Block diagram of health monitoring system

The values of temperature, blood pressure and heart beat sensors of the patient are input. The age of the patient is determined using potentiometer. These sensors are connected to Arduino mega 2560.All these values are then sent to Arduino mega to check with threshold values in simulation mode using proteus professional suite. Tested values will be displayed in virtual terminal. If the values are abnormal, Email will be sent to respective patient and the doctor.

\section{FLOWCHART}

The flow chart of the control system for data analytics approach of patient health monitoring is shown inFig.3.2.

Once Arduino super starts the operation, using virtual com, sensors and blynk cloud are initialized. Sensors then read the values of patient as per the potential meter variation. These values are checked with threshold values using Arduino mega. If the values surpass the threshold values, then the patient and the doctor will receive an e-mail warning. Then buzzer and
GPS will be activated to check the current location to locate the nearest health care center.

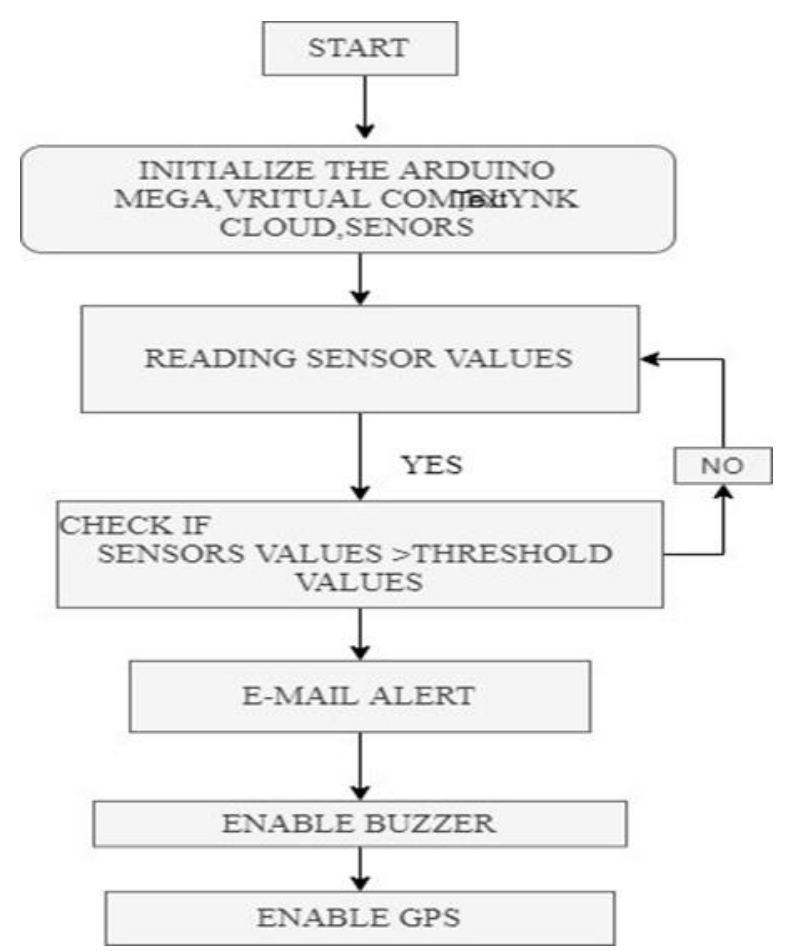

Fig3.2. Flow chart of health monitoring system.

\section{RESULTS}

The Model has been simulated by using Proteus Software to monitor the human health using different sensors with no limitations of age. After initialization of circuit all the values of temperature, blood pressure and heartbeat rate are inputted and age of the person through potentiometer. Fig 7.1 shows circuit diagram of health monitoring system and the values obtained in virtual terminal. Fig7.2 shows the tested values of sensors in blynk app. All the tested values are displayed in blynk app along with current location which is useful to locate the patient to the nearby health care center. As the health parameters are beyond the threshold Values, the person is notified through mail. At the same time buzzer also reached the $\mathrm{ON}$ state to make the person realize the condition. 


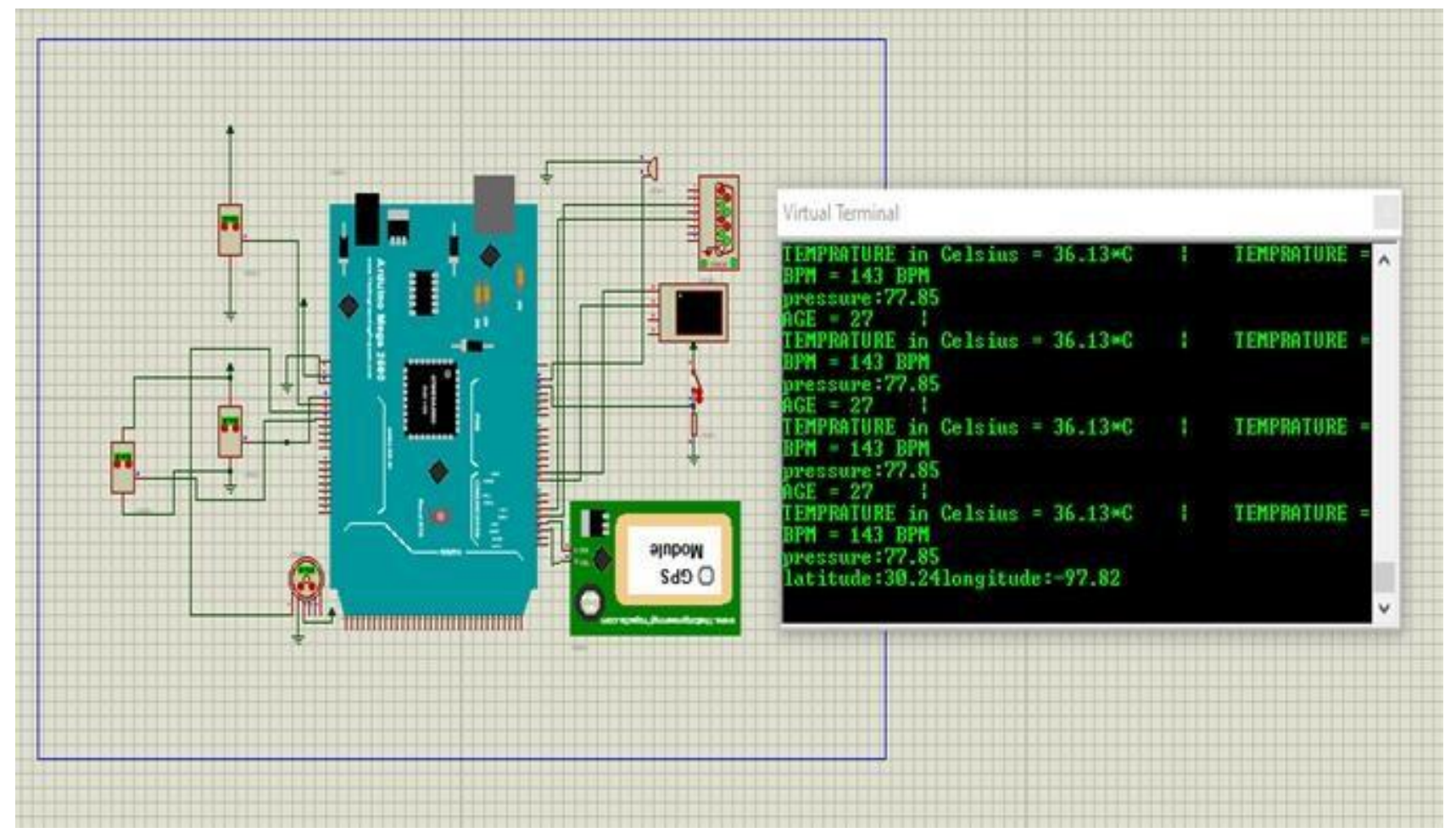

Fig7.1 Circuit diagram of health monitoring system

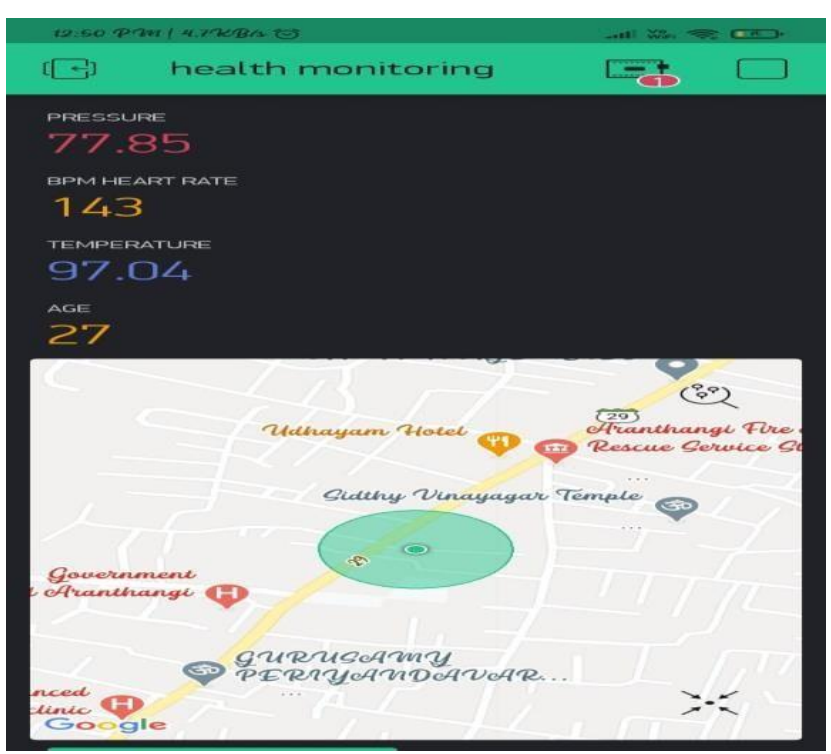

Fig7.2Tested values in blynk app.

Fig7.3 shows the E-mail received by the patient. As the sensor values have crossed the threshold values an alert has been sent to patient as E-mail along with the parameter store at it accordingly.

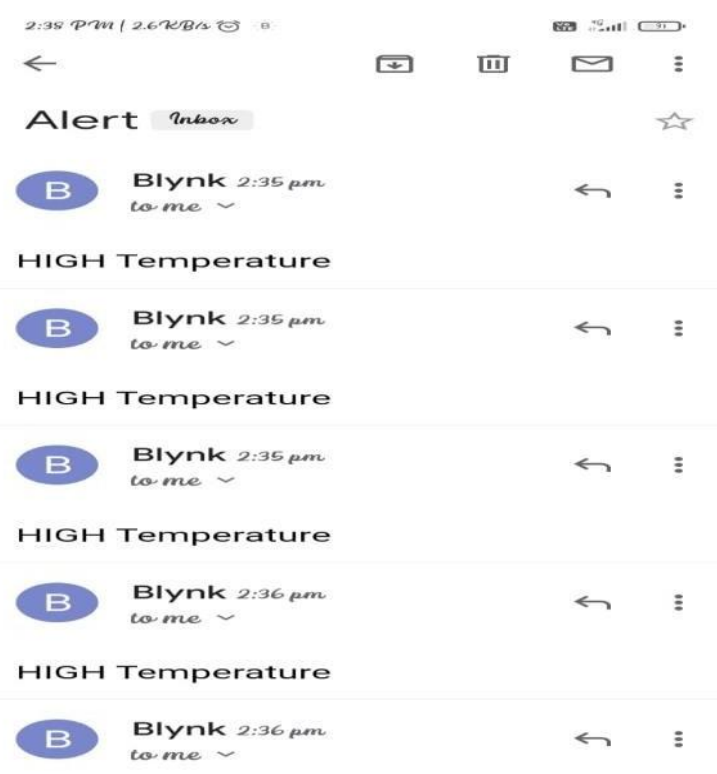

Fig7.3E-mail received by patient.

By making data analytics approach for patient's data with different age groups using weka tool the followings graphs are obtained along with the errors in pressure and threshold values. Fig 7.4 shows the blood pressure prediction graph. 


\begin{tabular}{|lr|}
\hline BP & \\
N & 71 \\
Mean absolute error & 5.4873 \\
Root relative squared error & 2491.4003 \\
Direction accuracy & 40 \\
Relative absolute error & 2904.9529 \\
Mean absolute percentage error & 6.2838 \\
Root mean squared error & 6.9467 \\
Mean squared error & 48.2568 \\
\hline & \\
\hline THRESHoLD vaLuES & 71 \\
N & 1.5669 \\
Mean absolute error & 302.8956 \\
Root relative squared error & 0 \\
Direction accuracy & 1083.712 \\
Relative absolute error & 1.2392 \\
Mean absolute percentage error & 2.1837 \\
Root mean squared error & 4.7685 \\
Mean squared error & \\
Total number of instances: 83 & \\
\hline
\end{tabular}

\section{Hot Spot}

Total population: 118 instances

Target attribute: THRESHOLD VALUES

Target average in total population: 127.364

Minimum segment size: 39 instances (33\% of total population)

Maximum branching factor: 2

Maximum rule length: unbounded

Minimum improvement in target: $1 \%$

THRESHOLD VALUES $(127.3644)$

$\mathrm{BP}>93.265(132.4615[39])$

$\mathrm{AGE}>30 \quad(128.8974 \quad[39])$

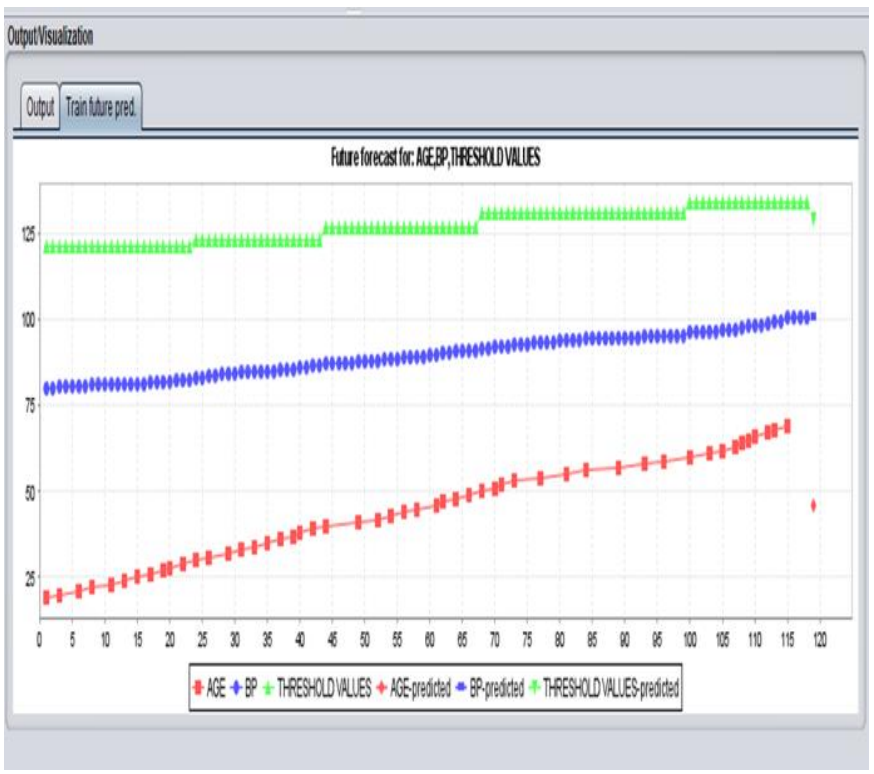

\section{Fig7.4Blood pressure predictiongraph}

Although low blood pressure (also known as "hypotension") is a less common issue than hypertension, it can also have a major effect on blood flow to the brain. Shock, stroke, heart attack, and kidney failure are all possibilities.

Fig 7.6 shows the heart rate prediction graph using weka tool with no limitations of age. Errors are also occurred while predicting the heart rate with its threshold values with respect to its concerned age.

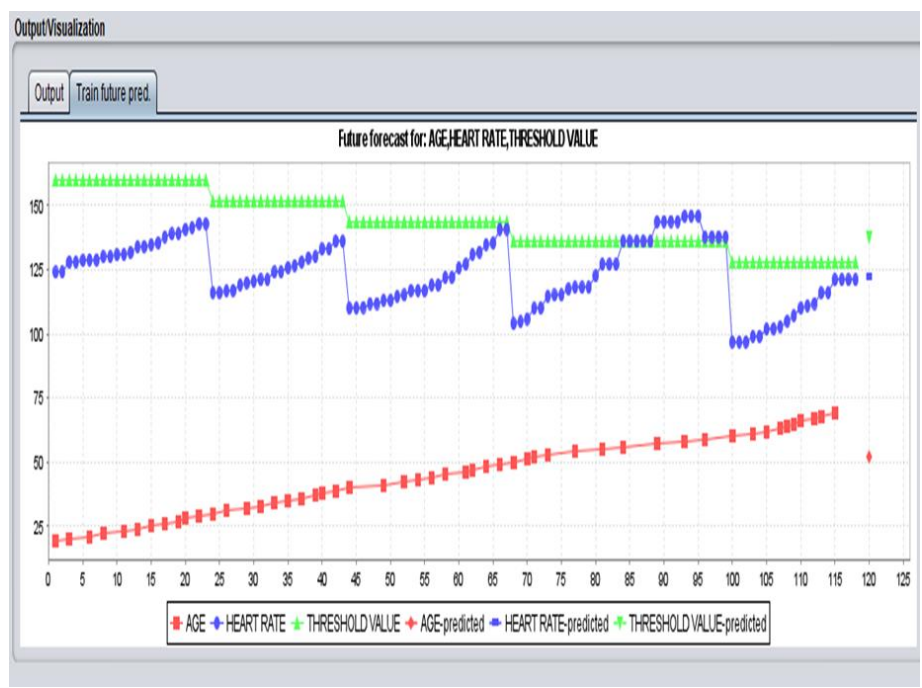

Fig7.6Heartrate prediction graph

The condition of the graph when the heart rate reaches the threshold values is shown in Fig 7.7. When your heart rate rises, you can experience the following symptoms: Shoulder, arm, and/or leg pain, numbness, and/or tingling Back, jaw, or back pain Shortness of breath can occur while you are active, at rest, or lying flat. You can feel chest pain during physical activity that subsides when you rest. I'm experiencing dizziness.

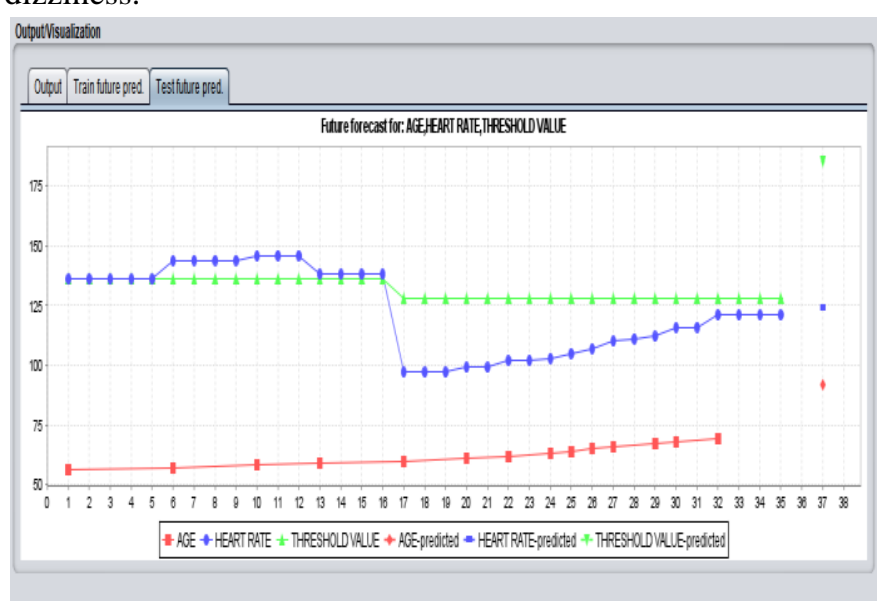

Fig7.7Heartrateexceededgraph

Fig 7.8 shows the temperature prediction graph of patient's 
temperature with no limitations of age Using weka tool. Certain error will also occur during the visualization.

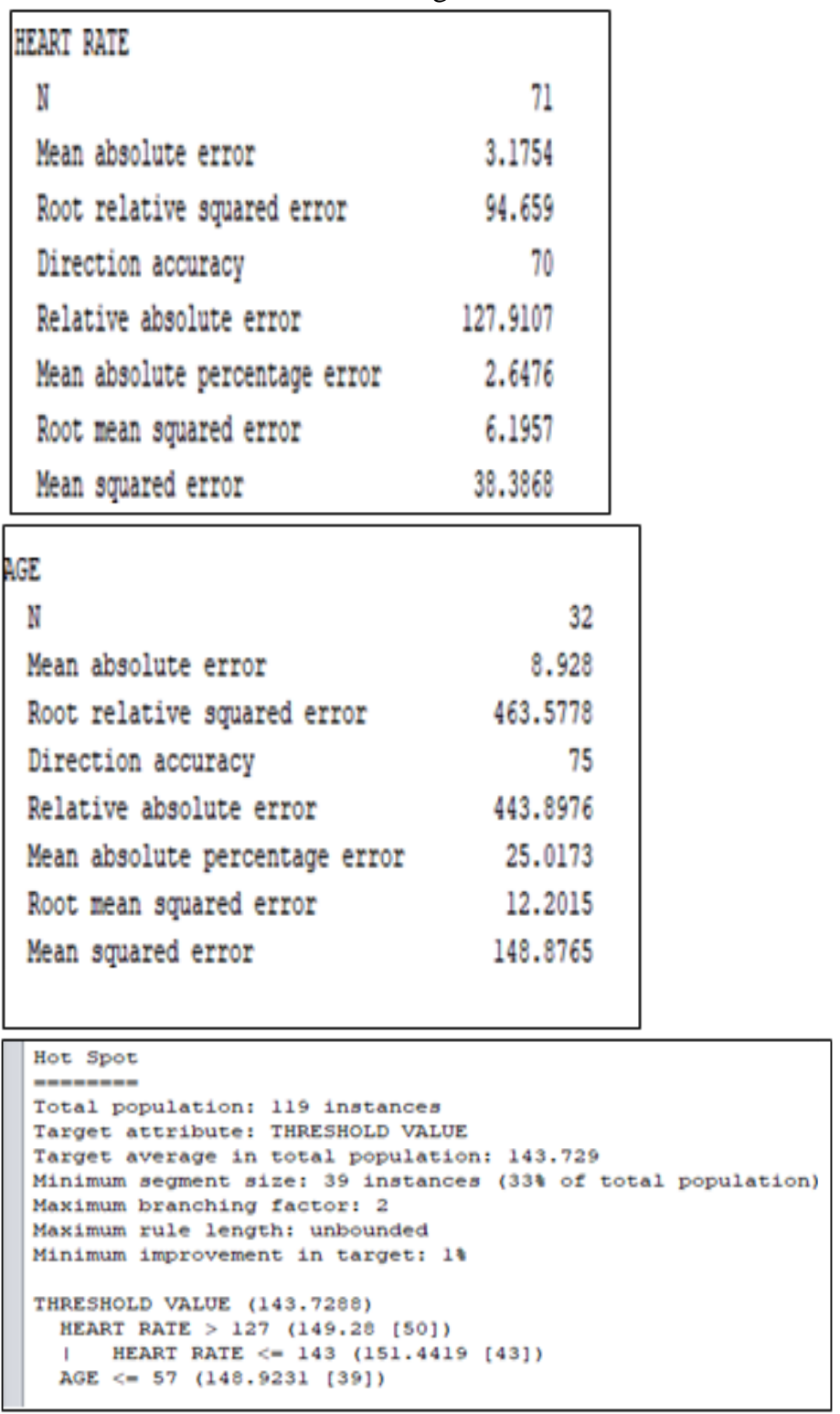

\begin{tabular}{|lr|}
\hline THRESHOLD VALUE & 71 \\
N & \\
Mean absolute error & 4.4395 \\
Root relative squared error & 394.1974 \\
Direction accuracy & 2.8571 \\
Relative absolute error & 1312.3436 \\
Mean absolute percentage error & 3.0769 \\
Root mean squared error & 6.4825 \\
Mean squared error & 42.0222 \\
& \\
Total number of instances: 83 & \\
\hline
\end{tabular}

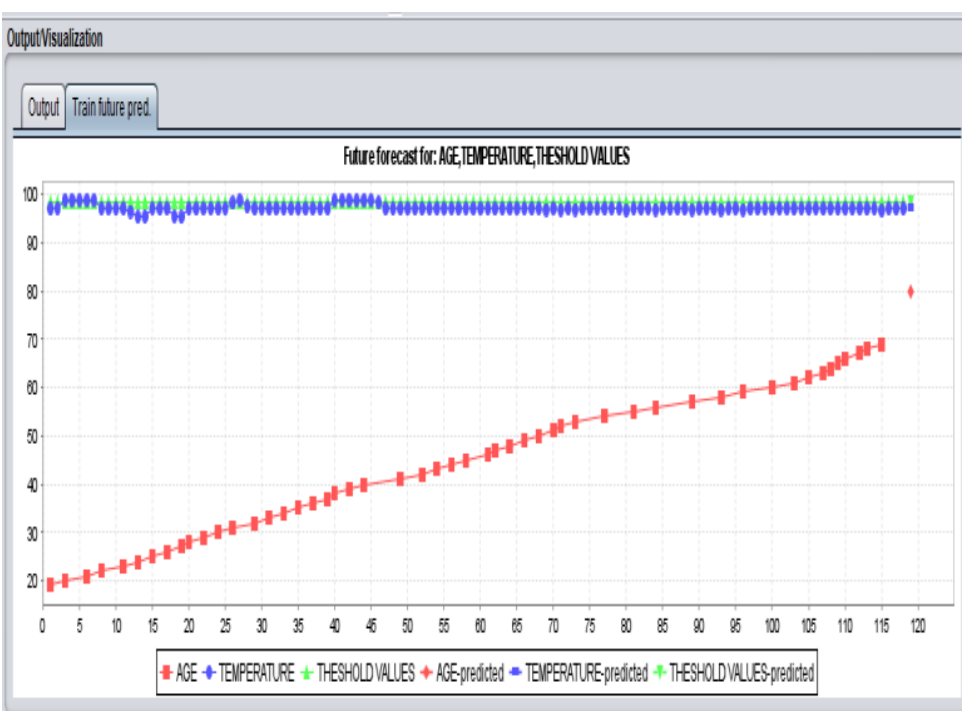

Fig7.8Temperature prediction graph

\begin{tabular}{|lr|}
\hline TEYPERATURE \\
$\mathbb{N}$ & 71 \\
Yean absolute error & 0.3305 \\
Root relative squared error & 97.1893 \\
Direction accuracy & 31.4286 \\
Relative absolute error & 143.5576 \\
Yean absolute percentage error & 0.3406 \\
Root mean squared error & 0.5102 \\
Yean squared error & 0.2603 \\
\hline
\end{tabular}

\begin{tabular}{|l|}
\hline THESHOLD VALUES \\
$\mathbb{N}$ \\
Nean absolute error \\
Root relative squared error \\
Direction accuracy \\
Relative absolute error \\
Nean absolute percentage error \\
Root mean squared error \\
Nean squared error \\
Total number of instances: 83 \\
Hot Spot \\
$=======$ \\
Total population: 118 instances \\
Target attribute: THESHOLD VALUES \\
Target average in total population: 98.6 \\
Minimum segment size: 39 instances (33\% of total population) \\
Maximum branching factor: 2 \\
Maximum rule length: unbounded \\
Minimum improvement in target: 18 \\
THESHOLD VALUES (98.6)
\end{tabular}




\section{CONCLUSION}

IoT is now widely regarded as one of the most viable options for remote data collection, especially in the field of health monitoring. Body temperature, heart rate and blood pressure were calculated by the device, which are also seen in the virtual terminal of proteus software. These sensor values are then sent to the cloud via wireless communication, where doctors and patients can access the information. In an emergency, the doctor will be alerted as well. The patient will then diagnose the disease and know about their health status based on the values received. Data analytics approach enhances the system by making patient's health condition to come to health care center's knowledge. In future even life insurance Company can also take this approach to make patients ensure their policy effectively.

\section{REFERENCES}

[1] Muhammad Sajjad, Shahryar Shafique Qureshi ,Muhammad Afnan, Department of Electrical Engineering, Iqra National University, Peshawar, Pakistan, IOT BASED HEALTH MONITORING SYSTEM USING ARDUINO UNO, GSM SIM900D MODULE AND WIFI MODULE ESP8266, published in Dec2018 by International Journal of Scientific \& Engineering Research(IJSER)

[2] PrajoonaValsalan,Tariq Ahmed Barham Baomar, Ali Hussain Omar Baabood Department of Electrical and Computer Engineering, Dhofar University, Salalah, Sultanate of Oman, IOT BASED HEALTH MONITORING SYSTEM, Vol 7, Issue 4, 2020,JournalofCritical Reviews

[3] Yedukondalu Udara, Srinivasarao Udara, Harish $\mathrm{H}$ M,Hadimani $\mathrm{H}$, Electronics and Communication Department, STJ Institute of Technology Ranebennur, 581115, India, HEALTH MONITORING SYSTEM USING IOT, International Journal of Engineering and Manufacturing Science,ISSN2249-3115Volume8, Number1 (2018) pp.177-182.

[4] Xiao Ma, Zie Wang, Sheng Zhou,Haoyu Wen, and Yin Zhang, School of Information and Safety Engineering, Zhongnan University of Economics and Law, Wuhan, China ,State Key Laboratory for Novel Sofware Technology, Nanjing University, China, Intelligent Healthcare Systems Assisted by Data analytics and Mobile Computing, Published in 3 July2018

[5] HadiBanaee , MobyenUddin Ahmed and AmyLoutfi,CenterforAppliedAutonomousSensorSystems ,OrebroUniversity,SE-70182Orebro,Sweden, Data Mining for Wearable Sensors in Health Monitoring Systems: A Review of Recent Trends and Challenges, Published in 17 December2013

[6] Jorge Gómez, ByronO viedob, Emilio Zhumab, Universidadde Córdoba,Departament ode Ingeniería
[7] De Sistemas, Montería-Colombia ,Universidad Técnica Estatal de Quevedo, Facultad de Ciencias dela Ingeniería, Quevedo - Ecuador, Patient Monitoring System Based on Internet of Things, published by Elsvier.B.V in The 7th International Conference onAmbient Systems, Networks and Technologies(ANT2016)

[8] YonglinRen, Richard Werner Nelem Pazzi, and Azzedine Boukerche, para diserese archlaboratory, University of Ottawa, Monitoring patients via a secure and mobile healthcare system, IEEE WirelessCommunications, February2010

[9] Padmashree.T, Dr.N.K.Cauvery ,Anirudh. V.C,Punith Kumar, Department of Information Science and Engineering, R.V.College of Engineering, Bengaluru, Patient Health Monitoring System and Prediction using Data Analytics, International Journal of Innovations in Engineering and Technology(IJIET)

[10] C.Senthamilarasi,J.JansiRani,B.Vidhya,H.Aritha,Electro nicsandCommunicationEngineering, Sri Krishna College of Engineering and Technology, Coimbatore, India, A SMART PATIENT HEALTH MONITORING SYSTEM USING IOT, International Journal of Pure and Applied Mathematics Volume 119 No. 16 2018, 59-70

[11] C.Premalatha, R.P.Keerthana, R.Abarna, Department of Information Technology, Sri Ramakrishna Engineering College, Coimbatore, India, human health monitoring system, published in Jan 2019 by International Research Journal of Engineering and Technology (IRJET),

[12] Shivkumar Dharmoji, Akshata Anigolkar, Prof.Shraddha M,Electronics and Communication Department, Jain College of Engineering, Belagavi, India, IoT based Patient Health Monitoring using ESP8266, International Research Journal of Engineering and Technology (IRJET), Volume: 07Issue:03, PublishedinMarch2020.

[13] D.ShivaRamaKrishnan1,SubhashChandGupta1,

Tanupriya Choudhury2, 1Amity University, UttarPradesh, Noida, India, 2 University of Petroleum and Energy Studies, Dehradun, India, An IOT based

[14] Health monitoring system published in 2018 International Conference on Advances in Computing and Communication Engineering (ICACCE-2018), by IEEE.

[15] Ayman Mir, Sudhir N.Dhage, Department of Computer Engineering Sardar Patel Institute of Technology Diabetes Disease Pre dictionusing Machine Learning on Big Data of Healthcare, published in 2018 by IEEE.

[16] Md. Raseduzzaman Ruman, Amit Barua, Waladur Rahman, Khan Roushan Jahan, Md.Jamil Roni, Md.Foyjur Rahman, IoT Based Emergency Health Monitoring System, published in 2020 International Conference on Industry 4.0 Technology by IEEE

[17] Age-Predicted Maxima lHeart Rate Revisited Hirofumi Tanaka, PHD, KevinD. Monahan, MS,Douglas R.Seals, PHD Boulderand Denver, Colorado, Journal of the American 
College of Cardiology, ( 2001bythe American College of Cardiology, published by Elsevier Science. 